\title{
SPIN MANIFOLDS ARE DECOMPOSABLE
}

\author{
R.E. STONG ${ }^{1}$
}

\begin{abstract}
It is shown that in the unoriented cobordism ring every manifold
\end{abstract} with Spin structure is decomposable.

1. Introduction. From the work of Thom [3], the unoriented cobordism ring $\mathfrak{R}_{*}$ is a polynomial ring over $Z_{2}$ on generators $x_{i}$ of dimension $i$, with $i \neq 2^{s}-1$, and the manifold $M^{i}$ is indecomposable if and only if the characteristic number $S_{i}\left[M^{i}\right]$ is nonzero.

Dold [2] exhibited odd dimensional manifolds which are suitable generators, and these generators are in fact orientable manifolds. No even dimensional oriented manifold can be indecomposable, since

$$
S_{2 n}\left[M^{2 n}\right]=\mathrm{Sq}^{1} S_{2 n-1}\left[M^{2 n}\right]=w_{1} S_{2 n-1}\left[M^{2 n}\right] .
$$

An examination of the known examples for generators quickly reveals that none admit Spin structures. This is not a coincidence, for one has

Proposition. Every Spin manifold is decomposable.

2. Proof. Since a Spin manifold is oriented, even dimensional Spin manifolds are decomposable. Further, if $M$ is a Spin manifold of dimension $4 n+1$,

$$
S_{4 n+1}[M]=\mathrm{Sq}^{2} S_{4 n-1}\lfloor M]=v_{2} S_{4 n-1}[M]=0 .
$$

Now, let $M$ be a Spin manifold of dimension $4 n+3$.

Claim. If $j \leqslant n$, and $x \in H^{n-j}\left(M ; Z_{2}\right)$, then $x^{4} S_{4 j+3}[M]=0$. This is clear for $j=0$, since $S_{3}$ is zero, and inductively it may be assumed if $j^{\prime}<j$.

Now $\mathrm{Sq}^{1} S_{4 j+3}=S_{4 j+4}=S_{2 j+2}^{2}=\operatorname{Sq}^{1}\left(S_{2 j+2} S_{2 j+1}\right)$, and by Proposition 6.1 of [1], $\mathrm{ker} \mathrm{Sq}^{1}=\mathrm{im} \mathrm{Sq}{ }^{1}$ in $H^{*}\left(B\right.$ Spin; $\left.Z_{2}\right)$ except in dimensions divisible by four. Thus $S_{4 j+3}=S_{2 j+2} S_{2 j+1}+\mathrm{Sq}^{1} \sigma$ for some $\sigma$, and

$$
x^{4} S_{4 j+3}[M]=x^{4} S_{2 j+2} S_{2 j+1}[M]+\mathrm{Sq}^{1}\left(x^{4} \sigma\right)[M]=x^{4} S_{2 j+2} S_{2 j+1}[M] .
$$

If $j$ is odd, $2 j+1=4 j^{\prime}+3, j^{\prime} \leqslant j$ and

$$
x^{4} S_{2 j+2} S_{2 j+1}[M]=\left(x S_{(j+1) / 2}\right)^{4} S_{4 j^{\prime}+3}[M]=0 .
$$

If $j$ is even,

Received by the editors July 6, 1976.

AMS (MOS) subject classifications (1970). Primary 57D75.

Key words and phrases. Cobordism, Spin manifold.

${ }^{1}$ The author is indebted to the National Science Foundation for financial support during this work.

๑ American Mathematical Society 1977 


$$
\begin{aligned}
x^{4} S_{2 j+2} S_{2 j+1}[M] & =x^{4} S_{2 j+2} \mathrm{Sq}^{2} S_{2 j-1}[M] \\
& =x^{4} \mathrm{Sq}^{2}\left(S_{2 j+2}\right) S_{2 j-1}[M] \\
& =x^{4} S_{2 j+4} S_{2 j-1}[M] \\
& =\left(x S_{(j+1) / 2}\right)^{4} S_{2 j-1}[M],
\end{aligned}
$$

which is zero, since $2 j-1=4 j^{\prime}+3, j^{\prime}<j$.

\section{REFERENCES}

1. D.W. Anderson, E.H. Brown, Jr. and F.P. Peterson, The structure of the Spin cobordism ring, Ann. of Math. (2) 86 (1967), 271-298. MR 36\#2160.

2. A. Dold, Erzeugende der Thomschen Algebra $\Re$, Math. Z. 65 (1956), 25-35. MR 18, 60.

3. R. Thom, Quelque propriétés globales des variétés différentiables, Comment. Math. Helv. 28 (1954), 17-86. MR 15,890.

Department of Mathematics, University of Virginia, Charlottesville, Virginia 22901 